\title{
LA LÍNEA DE MOBILIARIO DE OFICINA OPENEST DE PATRICIA URQUIOLA Y LA CREACIÓN DE NUEVOS ESPACIOS DE TRABAJO THE OPENEST COLLECTION BY PATRICIA URQUIOLA AND THE CREATION OF TODAY'S WORK ENVIROMENTS
}

Alicia Menéndez Martínez* Universidad de Oviedo

Resumen

Con la colección Openest para Haworth Patricia Urquiola abrió en 2014 una nueva línea de investigación sobre el diseño de mobiliario de oficina y la proyección de ambientes laborales en la que aún hoy sigue trabajando. Este encargo supuso para la diseñadora el estudio y reflexión sobre la evolución a lo largo de la historia de los espacios de trabajo y la creación de soluciones para afrontar las necesidades del futuro determinadas por las nuevas tecnologías.

Palabras clave: Mobiliario, oficina, diseño, arquitectura, Haworth, Openest, Patricia Urquiola.

\section{Abstract}

In 2014 Patricia Urquiola focused her research on office interiors and furniture solutions with the Openest collection for Haworth. Today, she is still investigating on new ways of working. This assignment took her to the study and reflection on the evolution of the office environment throughout the history and the creation of solutions to face future needs determined by new technologies.

Keywords: Furniture, office, design, architecture, Haworth, Openest, Patricia Urquiola.

\footnotetext{
*E-mail: aliciamenendez@hotmail.es
} 
En 2014 se presentaba al público la línea Openest de la diseñadora y arquitecta española Patricia Urquiola (Oviedo, 1961) en Orgatec, la feria especializada en mobiliario de oficina de Colonia (Alemania). Lo que podría parecer una presentación de otro nuevo producto en la ya exitosa carrera de la diseñadora entrañaba un hito más en su trayectoria. La colección Openest supuso su irrupción en el mercado estadounidense de la mano de la firma Haworth y la apertura de un campo de investigación nuevo para Urquiola sobre la evolución, transformación y futuro de los espacios de trabajo.

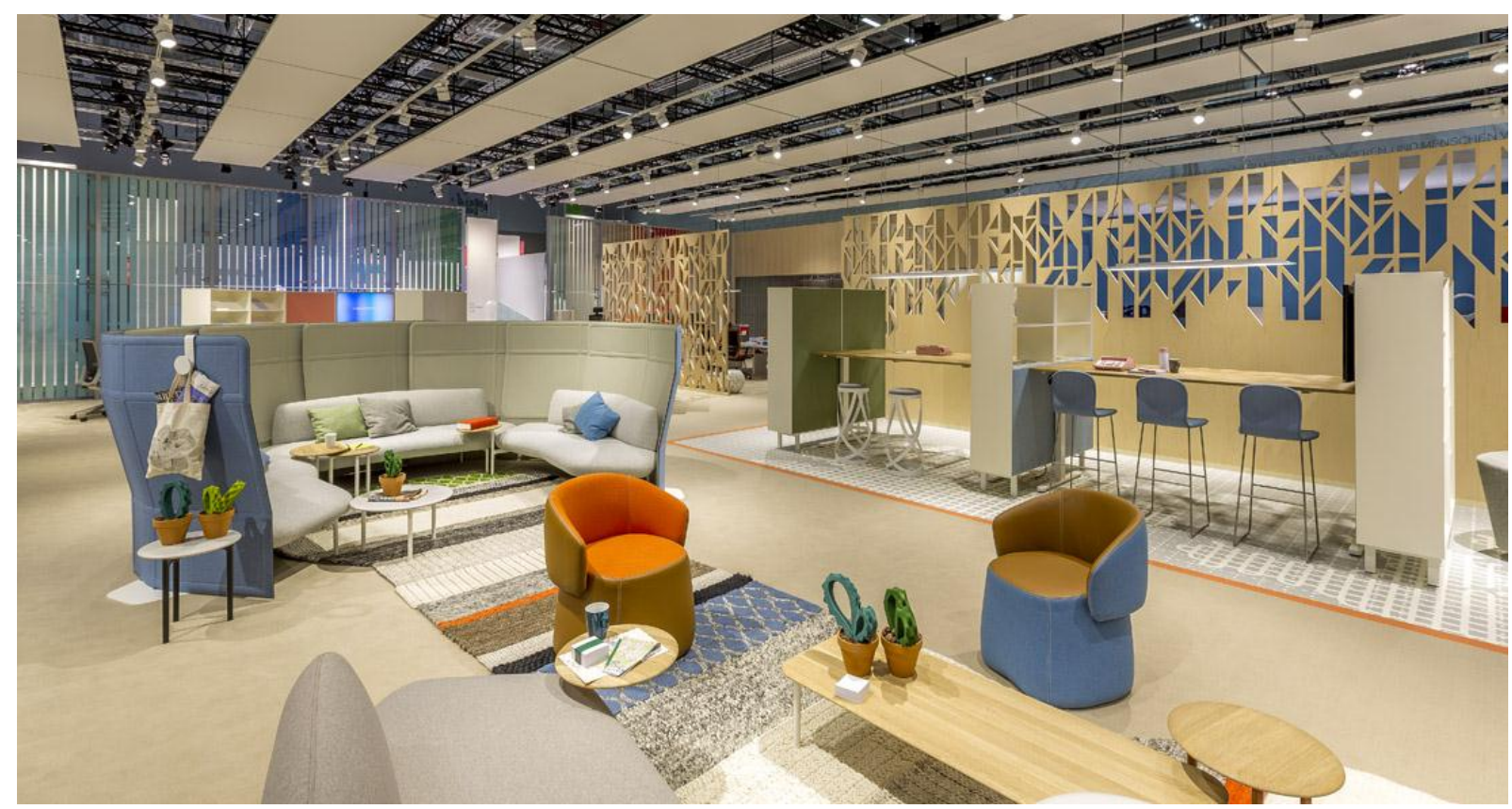

Fig. 1. Presentación del mobiliario y espacios de trabajo Openest para Haworth en la feria Orgatec de Colonia (Alemania, 2014).

Flexibilidad, libertad, calidez, comunicación e inspiración son algunos de los conceptos de los que se valen los diseñadores del presente para plantear los ambientes laborales del siglo XXI. Las innovaciones introducidas en este ámbito por arquitectos como Louis Sullivan y Frank Lloyd Wright que se vieron plasmadas a principios del siglo pasado en edificios de oficinas como el Larkin de Búfalo ${ }^{1}$, siguieron vigentes durante décadas. Wright había apostado en el mencionado edificio por la apertura de un espacio diáfano en detrimento de las estancias cerradas y compartimentadas, proyectando una sólida base sobre la que iría evolucionando el interiorismo de oficina durante décadas. Como señala Nikil Saval en su obra Cubed. A secret history of the workplace ${ }^{2}$, en los años sesenta los proyectistas, ayudados por nuevas herramientas tecnológicas, empezaron a imaginar nuevos espacios que dieran cabida al enorme flujo de papel que generaban los 'oficinistas' en su rutina diaria y con ellos, nuevas soluciones.

La atención prestada a la mejora de los ambientes laborales no ha cesado desde entonces. Quizá la evolución más notable se haya producido, como también señala Saval, durante los años noventa propiciada por la explosión y expansión de internet y toda una serie de firmas relacionadas con la red que él define como 
"burbuja.com" ("dot-com bubble"). ${ }^{3}$ Esta revolución tecnológica y los responsables de la misma trastornan la rigidez de las oficinas clásicas que ya no responden a las necesidades de unos trabajadores inmersos en una nueva era: la era digital. Los profesionales del diseño y la arquitectura afrontaron, a partir de ese momento, un reto importante. Tenían el deber de actuar como visionarios a la manera de Sullivan y Wright para proyectar nuevos espacios orgánicos y de confort en los que mejorar la satisfacción de los trabajadores, ya que en ese contento se hallaría un mayor rendimiento y un aumento de su creatividad y de su producción. Nacía así una nueva filosofía que superaba para siempre el taylorismo ${ }^{4}$ y otros intentos de organización del trabajo. El siglo XX finalizaba con una atención especial a la felicidad y confortabilidad del trabajador dentro del espacio en el que desempeña sus tareas diariamente.

Es responsabilidad, por tanto, de las firmas especializadas en el diseño de los lugares de trabajo de esta centuria ofrecer soluciones felices para empresas y trabajadores satisfechos. Algunas de ellas, como la estadounidense Haworth ${ }^{5}$, fundada por G. W. Haworth en 1948, están embarcadas en la tarea de hacer evolucionar el concepto clásico de oficina y de reinventarse a sí mismas para dar soluciones acordes a estos tiempos. Con este objetivo han apostado por el talento de profesionales que desde distintas partes del mundo han aportado sus ideas para dar un 'giro copernicano' a los antiguos planteamientos.

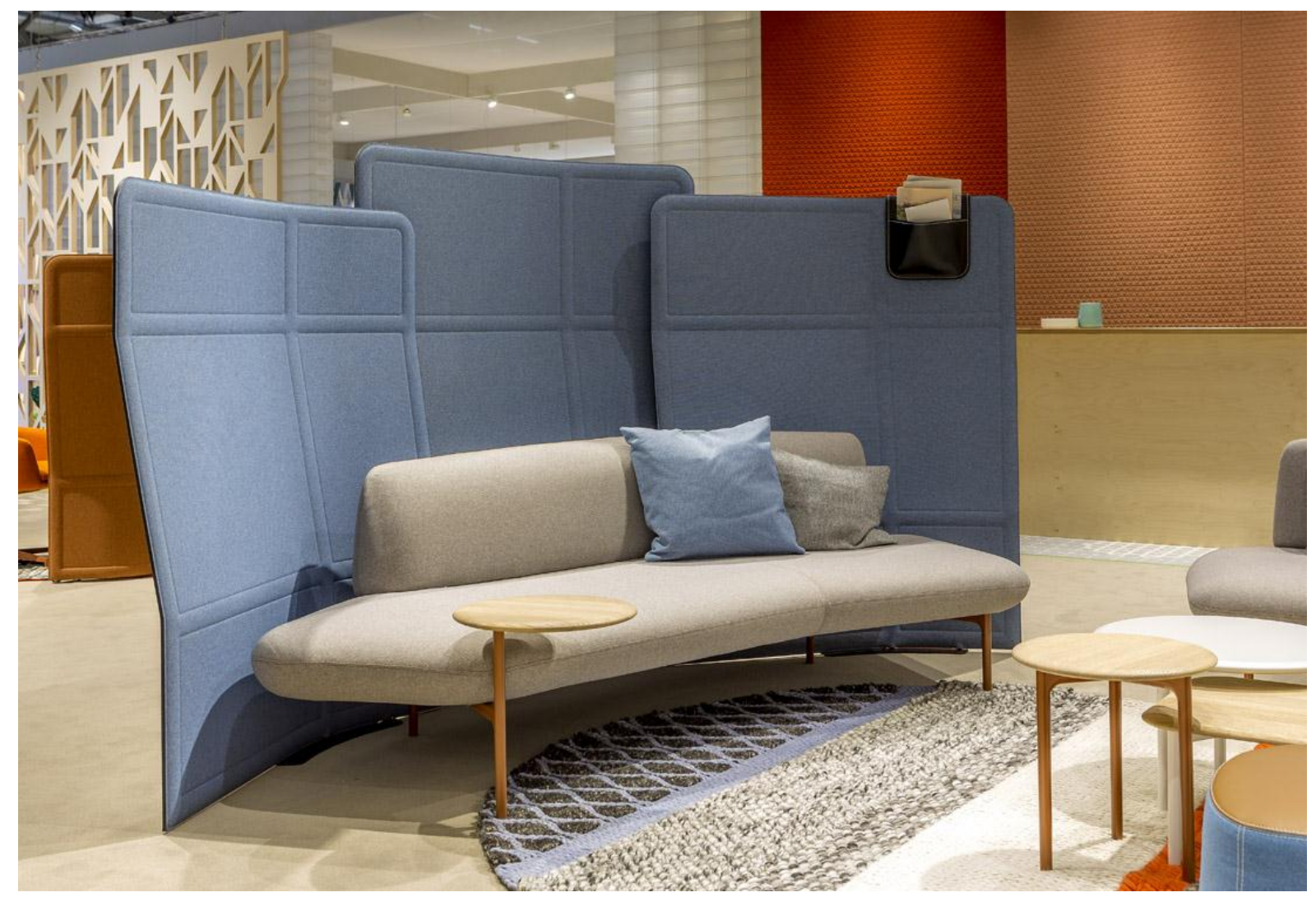

Fig. 2. Uno de los espacios Openest creados por Patricia Urquiola. Aparecen sus diseños de mampara (Plume) y asientos (Feather) para Haworth, en los que ha sumado su diseño de alfombra Mangas para la firma española Gan con el propósito de dotar a este espacio de calidez y confortabilidad. 
Haworth ha pasado de ofrecer en sus catálogos equipamientos de oficina rígidos y clásicos a poner a disposición del cliente proyectos integrales "de suelo a techo y de pared a pared" ("from floor to ceiling, and wall to wall”), según reza en su página web 6 , apostando por la tecnología Workware ${ }^{7}$, específica para ellos, que ofrece soluciones de conectividad para integrar el mobiliario con las últimas tecnologías necesarias en el entorno laboral. Para la parte más creativa recurrieron a Patricia Urquiola, quien expuso al público sus primeras ideas para la marca en la mencionada feria de Colonia en 2014, tras dos años de investigación y conversaciones con sus responsables.

Urquiola supuso un cambio radical para una compañía que facturaba 1,4 billones de dólares gracias a sus ventas de productos y soluciones destinadas a oficinas a gran escala, según un dato recogido en la revista especializada en diseño Wallpaper ${ }^{8}$. Este nuevo enfoque para Haworth se materializó en Openest, un diseño concebido para fomentar la comunicación y la colaboración en equipos reducidos de trabajo. Con ello, Urquiola aportaba una escala europea a los siempre amplios y sobredimensionados espacios americanos. Así, la diseñadora restaba rigidez y formalidad e introducía espontaneidad gracias a la flexibilidad de un diseño como Openest que ofrece una gran variedad de combinaciones posibles.

La flexibilidad es una constante en la producción de la diseñadora, pues la encontramos en la mayoría de su mobiliario doméstico, donde un mismo sofá, como ejemplo, presenta una variedad de opciones que obliga al cliente a tomar decisiones en cuanto a las formas (combinación a la carta de distintos elementos modulares), texturas (en los revestimientos) y colores personalizando así el diseño en cierta medida. En esta ocasión, Urquiola introduce además en Openest dos conceptos ajenos por lo general a la 'asepsia' que se presupone en un lugar de trabajo como son el confort y la domesticidad.

La colección de sofás, pufs, mesas y pantallas divisorias que conforman esta línea de Urquiola para Haworth transmiten una calidez propia de un espacio doméstico, invitando por tanto a la reflexión y a los encuentros informales "cara a cara" de los miembros de un mismo equipo. "When people come to work, they need to feel good" ("cuando la gente viene a trabajar, necesita sentirse bien"), afirmaba Patricia Urquiola en unas declaraciones para la revista especializada en diseño Wallpaper $^{9}$. Esta es la filosofía que se esconde tras este diseño, pero también detrás de la oficina del futuro que, como algunos vaticinan -el propio Saval ${ }^{10}$, entre ellos- está abocada a la desaparición como espacio físico específico para ser reemplazada por una oficina virtual que solo necesite espacios más informales, similares a salas de estar y cafés, donde puedan intercambiarse impresiones en persona.

En la propuesta de interiorismo Openest los tabiques no existen. Han sido sustituidos por pantallas divisorias llamadas Plume que actúan como elementos conformadores de espacios más íntimos dentro de otros más diáfanos. La altura de estas mamparas portátiles, así como sus formas envolventes y curvas organizan los espacios, mientras que la delicada paleta de color en la que se 
presenta su revestimiento y su suave textura, transmiten por sí solas la sensación de confort que persigue la diseñadora.

Existen asimismo en Openest los Conference booth o cubículos pensados para multi-conferencias que dan cobijo a un reducido número de personas, normalmente no más de cuatro, con acceso a los medios tecnológicos necesarios para el trabajo colaborativo y la interconexión simultánea.

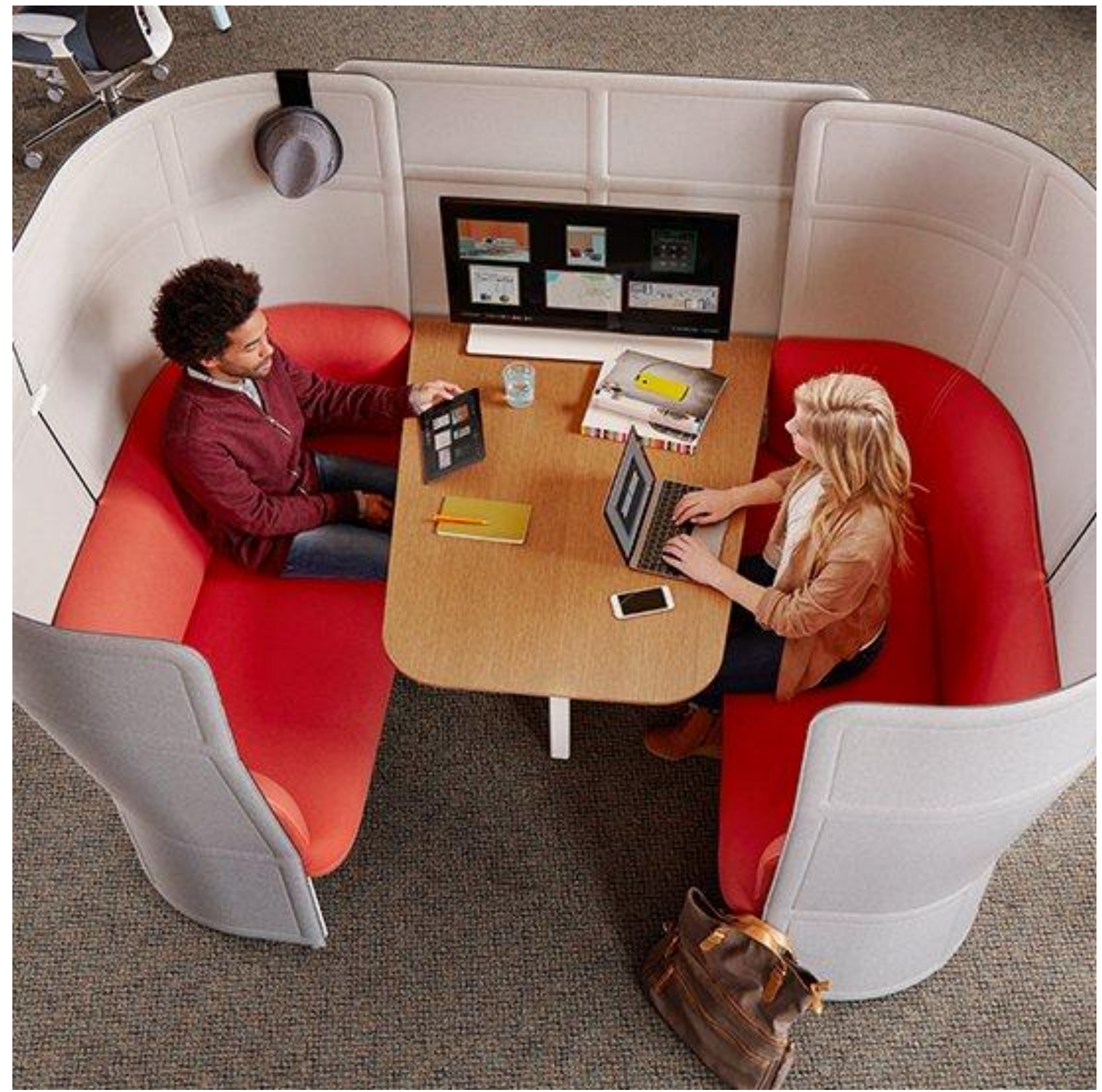

Fig. 3. Imagen de uno de los Conference booth, que actúan como mini-despachos transformables.

Patricia Urquiola muestra, así, sus ideas sobre un campo que ella considera en permanente evolución y aplica de nuevo todos sus conocimientos sobre técnicas, tecnologías, materiales, etc., lo que le ha valido el reconocimiento en ferias internacionales especializadas en espacios de trabajo como la NeoCon de 
Chicago (EE.UU.), donde el proyecto Openest fue galardonado con el Premio Gold for Systems y el Best of NeoCon, ambos en 2014, y los premios HiP de la misma feria en 2015 y 2016.

\section{NOTAS}

\footnotetext{
${ }^{1}$ El Edificio Larkin fue proyectado para la Larkin Soap Company por el arquitecto y diseñador estadounidense Frank Lloyd Wright en 1903 e inaugurado en 1906 en Búfalo (EE.UU.).

${ }^{2}$ Saval, Nikil, Cubed. A secret history of the workplace, Nueva York, Anchor Books, 2014.

${ }^{3}$ Ibídem, p. 5.

${ }^{4}$ Frederick Taylor (1856-1915) estudió de manera científica los procesos de producción ligados a la Revolución Industrial y planteó una organización del trabajo basada en la división de tareas y el aumento de la productividad.

${ }^{5}$ Cfr. http://www.haworth.com/home Última consulta: 29/11/2017.

${ }^{6}$ Cfr. http://www.haworth.com/company-info/about-us Última consulta: 29/11/2017.

${ }^{7} \mathrm{Cfr}$. http://www.haworth.com/products/technology/collaboration/workware Última consulta: 29/11/2017.

${ }^{8}$ Martin, J. J., "Patricia Urquiola's new 'Openest' office furniture line for Haworth", Wallpaper, 10 de junio de 2014.

${ }^{9}$ Ibídem.

${ }^{10}$ Saval, Nikil, Op. Cit.
}

Fecha de recepción: 04-12-2017

Fecha de revisión: 11-12-2017

Fecha de aceptación: 12-12-2017 